Marquette University

e-Publications@Marquette

$11-2017$

\title{
Investigating Habituation to Premonitory Urges in Behavior Therapy for Tic Disorders
}

\author{
David C. Houghton \\ Texas A\&M University - College Station \\ Matthew R. Capriotti \\ University of California, San Francisco \\ Lawrence Scahill \\ Emory University \\ Sabine Wilhelm \\ Harvard Medical School \\ Alan L. Peterson \\ University of Texas Health Science Center at San Antonio
}

See next page for additional authors

Follow this and additional works at: https://epublications.marquette.edu/psych_fac

Part of the Psychology Commons

\section{Recommended Citation}

Houghton, David C.; Capriotti, Matthew R.; Scahill, Lawrence; Wilhelm, Sabine; Peterson, Alan L.; Walkup, John T.; Piacentini, John; and Woods, Douglas W., "Investigating Habituation to Premonitory Urges in Behavior Therapy for Tic Disorders" (2017). Psychology Faculty Research and Publications. 285.

https://epublications.marquette.edu/psych_fac/285 


\section{Authors}

David C. Houghton, Matthew R. Capriotti, Lawrence Scahill, Sabine Wilhelm, Alan L. Peterson, John T. Walkup, John Piacentini, and Douglas W. Woods 
Marquette University

\section{e-Publications@Marquette}

\section{Psychology Faculty Research and Publications/College of Arts and Sciences}

This paper is NOT THE PUBLISHED VERSION; but the author's final, peer-reviewed manuscript. The published version may be accessed by following the link in the citation below.

Behavior Therapy, Vol. 48, No. 6 (2017): 834-846. DOI. This article is (C) Elsevier and permission has been granted for this version to appear in e-Publications@Marquette. Elsevier does not grant permission for this article to be further copied/distributed or hosted elsewhere without the express permission from Elsevier.

\section{Investigating Habituation to Premonitory Urges in Behavior Therapy for Tic Disorders}

David C. Houghton

Texas A\&M University

Matthew R. Capriotti

San Jose State University

University of California San Francisco

Lawrence D. Scahill

Emory University

Sabine Wilhelm

Massachusetts General Hospital/Harvard Medical School

Alan L. Peterson

University of Texas Health Science Center at San Antonio

John T. Walkup

Weill Cornell Medical College of Cornell University

John Piacentini

University of California Los Angeles

Douglas W. Woods

Marquette University 


\section{Abstract}

Behavior therapy is effective for Persistent Tic Disorders (PTDs), but behavioral processes facilitating tic reduction are not well understood. One process, habituation, is thought to create tic reduction through decreases in premonitory urge severity. The current study tested whether premonitory urges decreased in youth with PTDs $(N=126)$ and adults with PTDs $(N=122)$ who participated in parallel randomized clinical trials comparing behavior therapy to psychoeducation and supportive therapy (PST). Trends in premonitory urges, tic severity, and treatment outcome were analyzed according to the predictions of a habituation model, whereby urge severity would be expected to decrease in those who responded to behavior therapy. Although adults who responded to behavior therapy showed a significant trend of declining premonitory urge severity across treatment, results failed to demonstrate that behavior therapy specifically caused changes in premonitory urge severity. In addition, reductions in premonitory urge severity in those who responded to behavior therapy were significant greater than those who did not respond to behavior therapy but no different than those who responded or did not respond to PST. Children with PTDs failed to show any significant changes in premonitory urges. Reductions in premonitory urge severity did not mediate the relationship between treatment and outcome in either adults or children. These results cast doubt on the notion that habituation is the therapeutic process underlying the effectiveness of behavior therapy, which has immediate implications for the psychoeducation and therapeutic rationale presented in clinical practice. Moreover, there may be important developmental changes in premonitory urges in PTDs, and alternative models of therapeutic change warrant investigation.

\section{Keywords}

Tics, psychotherapy, behavior therapy, habituation

Persistent tic disorders (PTDs) such as Tourette's Disorder (also known as Tourette's syndrome) are neurodevelopmental disorders characterized by the presence of tics for at least 1 year (American Psychiatric Association, 2013). Tics are repetitive motor movements (e.g., hard blinking and head jerking) and vocalizations (e.g., grunting and repetition of words or phrases) that can cause significant functional impairment and distress (Houghton, Alexander, \& Woods, 2016). PTDs primarily affect children and have a waxing-to-waning developmental course. The age of tic onset tends to be between 4 and 6 years, and tics reach peak severity between ages 10-12 and often decline in severity during late adolescence (Bloch \& Leckman, 2009). However, nearly one quarter of individuals with PTDs experience chronic tic symptoms into adulthood (Bloch and Leckman, 2009, Groth et al., 2017, Leckman et al., 1998).

PTDs can be treated effectively with behavior therapy (Capriotti et al., 2014, Cook and Blacher, 2007, Piacentini et al., 2010, McGuire et al., 2014, Wilhelm et al., 2012), which is thought to facilitate conditioning experiences central to promoting tic reduction. Behavioral interventions approach tics as being initiated by aberrant neural functioning but perpetuated largely by conditioning processes surrounding core PTD symptoms (Conelea and Woods, 2008, Himle et al., 2006, Himle et al., 2014). Indeed, a neurobehavioral perspective on tics acknowledges that tics are supported by motor hyperexcitability within fronto-striatal neural circuits (Albin \& Mink, 2006), but tics are maintained, in part, by operant reinforcement and respondent associations (reviewed by Himle et al., 2006). One crucial aspect of these conditioning processes involves the functional relation between certain somatic 
phenomena, known as premonitory urges (PMUs), and tics (reviewed by Houghton, Capriotti, Conelea, \& Woods, 2014).

A substantial body of literature has shown that individuals with PTDs experience PMUs, which are aversive sensations that precede and accompany tics (Cohen and Leckman, 1992, Kurlan et al., 1989, Kwak et al., 2003, Leckman et al., 1994, Leckman et al., 1993, Woods et al., 2005). Patients describe these experiences as various feelings of unfulfillment, irritation, and musculoskeletal tension (Bliss, Cohen, \& Freedman, 1980). Whereas early conceptualizations considered tics to be involuntary (Caine, Polinsky, Kartzinel, \& Ebert, 1979), accounts of PMU phenomena suggested that tics are better characterized as somewhat volitional and instigated by highly aversive PMUs, which are alleviated upon ticcing (Evers and van de Wetering, 1994, Kane, 1994, Lang, 1991). Several studies have supported this notion using an experimental paradigm comparing periods in which tic suppression is intermittently reinforced by monetary reward with periods when participants are instructed to tic freely and suppression is not rewarded (e.g., Capriotti et al., 2014, Himle et al., 2007, Woods and Himle, 2004). Results of these studies showed that tics can be suppressed for brief periods and that PMU strength increased during reinforced tic suppression and decreased during breaks from suppression. Furthermore, a recent study found that PMU strength increases prior to ticcing and decreases after ticcing (Brandt, Beck, Sajin, Baaske, et al., 2016).

The neurobehavioral model of PTDs posits that the short-term reductions in PMUs following tic completion result in longer-term strengthening or maintenance of tics and PMUs (Himle et al., 2006). When individuals engage in prolonged tic suppression, they experience PMUs without ticcing, and PMUs are thought to dissipate (Woods et al., 2008). Thus, tic suppression might facilitate a PMU habituation process whereby repeated exposure to the PMU results in decreased physiological response to PMUs and similar sensory stimuli (Evers and van de Wetering, 1994, Himle et al., 2006, Hoogduin et al., 1997, Verdellen et al., 2008, Woods et al., 2000). PMU habituation is thought to occur both within individual periods of tic suppression (i.e., within treatment sessions) and between periods of suppression (i.e., across sessions). The notion of PMU habituation through tic suppression is similar to the rationale underlying exposure and response prevention (ERP) for obsessive-compulsive disorder (Abramowitz, 1996). However, ERP-based behavioral treatments for PTDs are thought to work by having clients engage in prolonged tic suppression and learn to habituate to the accompanying increases in PMUs (Verdellen, Keijsers, Cath, \& Hoogduin, 2004), whereas ERP for obsessivecompulsive disorder (OCD) involves deliberate exposure to fear-evoking stimuli and prevention of rituals and/or avoidance behaviors.

Studies investigating PMU habituation have yielded mixed results. The first study to systematically investigate PMU habituation reported on 4 patients with Tourette's Disorder ( 3 adults and 1 child) who received 10 sessions of ERP for PTDs (Hoogduin et al., 1997). Repeated-measures data from 3 of the 4 participants showed evidence of short-term PMU habituation within sessions (i.e., decreases in urge ratings at the end of a session relative to the beginning of those same sessions). In an open trial of 19 adults and children with PTDs who received the same ERP treatment protocol, longitudinal analyses showed evidence of PMU habituation both within and between sessions (Verdellen et al., 2008). However, three studies found a lack of evidence for within-session PMU habituation using differential reinforcement for tic suppression paradigms lasting 25-80 minutes (Capriotti et al., 2014, Himle et al., 2007, Specht et al., 2013).

These mixed findings may be at least partially due to methodological inconsistencies and shortcomings. First, studies examining PMU habituation have used samples of differing age ranges, despite the fact 
that there are important age-based differences in PMUs. Research has shown that while a majority of persons with PTDs aged 9 years or older report some type of PMU, younger children are less likely to report PMUs (Banaschewski et al., 2003, Leckman et al., 1993, Woods et al., 2005), suggesting younger children may show a less clear association between PMUs and tics throughout treatment. Indeed, studies that showed positive evidence of PMU habituation included both adults and children but were weighted toward adults (Hoogduin et al., 1997, Verdellen et al., 2008). For example, the sample in Verdellen et al. had a mean age of 23 , and in the study by Hoogduin et al., the three participants who showed evidence of PMU habituation were adults while the one participant showing no PMU habituation was a child. By comparison, the studies that reported a lack of evidence for PMU habituation used younger samples consisting of children and adolescents (Capriotti et al., 2014, Capriotti et al., 2014, Himle et al., 2007, Specht et al., 2013). These results suggest that perhaps adults but not children experience PMU habituation during behavioral treatment. A second limitation of existing evidence for or against the habituation hypothesis is that lab-based tic suppression studies, on which the findings primarily have been based, may not generalize to clinical settings and have used relatively small sample sizes with no control groups (Capriotti et al., 2014, Himle et al., 2007, Specht et al., 2013). As such, future research is needed to examine PMUs using (a) studies that enable differential analyses for adults and children, (b) longitudinal designs that reflect real-world behavior therapy for PTDs, and (c) large samples with control conditions.

The current study sought to examine PMU habituation as a mechanism of change within two, large, multi-site trials of behavior therapy, based primarily on habit reversal training (HRT; Azrin \& Nunn, 1973), versus supportive psychotherapy plus psychoeducation control for pediatric (Piacentini et al., 2010) and adult (Wilhelm et al., 2012) PTDs. Data from the child trial were analyzed separately from the adult trial in order to examine age-based differences. Several predictions were made to examine whether PMU habituation occurred. First, PMU severity should have decreased across time for those who received behavior therapy but not for those who received a control treatment (Hypothesis 1), particularly in those who responded to treatment (Hypothesis 2). It was also predicted that pre- to posttreatment decreases in PMU severity should mediate the relationship between treatment condition and outcome (Kraemer, Wilson, Fairburn, \& Agras, 2002) (Hypothesis 3). In addition, because some research has indicated that premonitory urges correlate positively with measures of OCD symptoms, anxiety, and depression (Eddy and Cavanna, 2013, Rozenman et al., 2015, Steinberg et al., 2009, Woods et al., 2005), exploratory analyses were conducted to determine whether reductions in PUTS scores were associated with change on any secondary outcome measures used in the clinical trials.

\section{Material and Methods}

\section{Participants}

In the child trial, a total of 178 children and adolescents were screened and 126 eligible youth enrolled between December 2004 and May 2007. Participants were recruited via psychiatry and psychology clinics, primary care and mental health referrals, schools, churches, community organizations, paid/public service notices, and ads in local media and on the Tourette Association of America website and newsletter. Inclusion criteria were (a) age 9-17 years, (b) Tourette's Disorder or Persistent Tic Disorder of at least moderate severity, (c) English language fluency, and (d) IQ > 80. Children who were medication free or on a stable medication regimen were eligible to participate. For a more detailed description of inclusion criteria and sample characteristics, see Piacentini et al. (2010) and Specht et al. 
(2011), respectively. Participants were randomized to receive the index treatment, Comprehensive Behavioral Intervention for Tics (CBIT), consisting of habit reversal training (HRT) plus functional intervention (Woods et al., 2008; $N=61$ ), or a comparison condition consisting of psychoeducation and supportive therapy (PST; $N=65$ ). By the end of the 10-week treatment phase, 5 participants (8\%) had discontinued behavior therapy, whereas 7 participants (11\%) discontinued supportive therapy.

In the adult trial, a total of 172 adults were screened and 122 eligible participants enrolled between December 2005 and May 2009. Participants were recruited via psychiatry and psychology clinics at major medical centers, flyers in public places, physician referrals, online advertisements, presentations at local patient organization meetings, and ads in local media. Inclusion criteria were identical to the child trial except that age was required to be $\geq 16$. Participants on stable medication for at least 6 weeks were allowed to participate. See Wilhelm et al. (2012) for a more detailed description of inclusion criteria and sample characteristics. Also similar to the child trial, participants were randomized to receive 10 weeks of CBIT $(N=63)$ or PST $(N=59)$. At the end of treatment, 7 participants (11\%) had discontinued behavior therapy, whereas 10 participants $(17 \%)$ discontinued supportive therapy.

\section{Assessments}

The Yale Global Tic Severity Scale (YGTSS) is a clinician-rated assessment of tic severity (Leckman et al., 1989). All current tics are rated on each dimension (score of $0-5$ ) for motor and vocal separately and then totaled for a score of $0-25$. The motor and vocal tic totals are summed for a combined total tic score (0-50). The YGTSS has adequate internal consistency (item-total correlations ranging from 0.780.88 ) and interrater reliability (intra-class correlation coefficients ranging from 0.52-0.99) and acceptable convergent and divergent validity (Leckman et al.).

The Premonitory Urge for Tics Scale (PUTS) is a self-report measure designed to assess PMU severity (Woods et al., 2005). The PUTS contains 9 items (displayed in Table 1) rated on a 4-point scale (1=not at all true to 4 = very much true). Item responses are summed for a score ranging from 9 (no PMUs) to 36 (high PMU severity). Internal consistency (Cronbach's alpha coefficients $=0.79-0.85$ ) and test-retest reliability has been reported in the acceptable to good range $(2$-week test-retest correlations $=0.79-$ 0.86), and concurrent validity of the PUTS is generally satisfactory (Crossley et al., 2014, Reese et al., 2014, Steinberg et al., 2009, Woods et al., 2005). A recent study also found that the PUTS showed convergent validity with real-time urge intensity scores on visual analogue scale (Brandt, Beck, Sajin, Anders, \& Munchau, 2016). However, studies have shown that the internal consistency and convergent validity of the PUTS is poorer in children younger than 10 as compared to youths older than 10 (Steinberg et al., 2009, Woods et al., 2005).

Table 1. Premonitory Urge for Tics Scale (PUTS)

\footnotetext{
Item

\#

Item Content

1 Right before I do a tic, I feel like my insides are itchy.

2 Right before I do a tic, I feel pressure inside my brain or body.

3 Right before I do a tic, I feel "wound up" or tense inside.

4 Right before I do a tic, I feel like something is not "just right"

5 Right before I do a tic, I feel like something isn't complete.
} 

Item
\#
Item Content
6 Right before I do a tic, I feel like there is energy in my body that needs to get out.
7 I have these feelings almost all the time before I do a tic.
8 These feelings happen for every tic I have.
After I do the tic, the itchiness, energy, pressure, tense feelings, or feelings that something isn't "just right" or complete go away, at least for a little while.

The Clinical Global Impressions-Improvement Scale (CGI-I; Guy \& Bonato, 1970) is a single-item clinician-rated measure of overall treatment response. A trained rater indicates improvement or worsening via an 8-point rating scale ranging from 1-8, with scores of "very much improved" (1) and "much improved" (2) defining treatment response. Reliability of the CGI-I has shown to be high in other disorders (i.e., schizophrenia; Cronbach's alpha $=0.69-0.96$ ) (Ventura, Cienfuegos, Boxer, \& Bilder, 2007). Validity coefficients are also high for the CGI-I across many different psychiatric conditions in both pharmacological and psychosocial treatment paradigms (Bandelow et al., 2006, Leon et al., 1993, Leucht and Engel, 2006, Spielmans and McFall, 2006, Zaider et al., 2003).

Several other self-report questionnaires were used to measure relevant secondary outcomes in the clinical trials. In the child trial, secondary outcome measures included the Children's Yale-Brown Obsessive-Compulsive Scale (Scahill et al., 1997), the attention problems subscale of the Child Behavior Checklist (Achenbach \& Edelbrock, 1991), the Disruptive Behavior Rating Scale-Parent Version (Friedman-Weieneth, Doctoroff, Harvey, \& Goldstein, 2009), the Child Depression Inventory (Helsel \& Matson, 1984), the Screen for Child Anxiety Related Emotional Disorders as rated by children and parents (Birmaher et al., 1997), and the Childhood Anxiety Sensitivity Index (Silverman, Fleisig, Rabian, \& Peterson, 2010). In the adult trial, secondary outcome measures included the Yale-Brown ObsessiveCompulsive Scale (Goodman et al., 1989), the Beck Anxiety Inventory (Beck, Epstein, et al., 1988), and the Beck Depression Inventory (Beck, Steer, \& Garbin, 1988).

\section{Procedure}

The child trial was conducted at three sites: The Johns Hopkins School of Medicine, the University of California, Los Angeles, and the University of Wisconsin-Milwaukee. The adult trial was conducted at three additional sites: Massachusetts General Hospital/Harvard Medical School, Yale University, and the University of Texas Health Science Center at San Antonio. Each of these sites also provided collaborative support in the form of administrative procedures, data management, rater training, and quality assurance across the two studies. Archival data analysis related to the present study was performed at Texas A\&M University. All institutions obtained IRB approval for the project, procedures were performed in compliance with the Code of Ethics of the World Medical Association (Declaration of Helsinki), and the studies are publicly listed on the U.S. National Institutes of Health human subjects trial forum (ClinicalTrials.gov; NCT00218777, NCT00231985). All adult participants and parents of child participants provided written informed consent, and child participants provided assent.

Participants in both trials completed 8 sessions of treatment across 10 weeks. CBIT consisted primarily of HRT (Azrin \& Nunn, 1973) but also included psychoeducation, relaxation training, and a functional intervention aimed at mitigating tic triggers (e.g., anxiety, public performance) and consequences associated with increased ticcing (e.g., teasing, escape from responsibility). The control treatment, PST, consisted of psychoeducation and supportive psychotherapy (Goetz \& Horn, 2005). PST precluded any 
instruction or advice pertaining to tic management strategies. Treatment conditions were matched in terms of time and therapist contact. For more detailed descriptions of therapeutic components, see Piacentini et al. (2010), Wilhelm et al. (2012), and Woods et al. (2008).

The current study utilized assessment data, collected from participants' self-reports, parent-reports, as well as trained clinical evaluators masked to treatment condition, from three time points: baseline $(0$ weeks), midtreatment (5 weeks), and posttreatment (10 weeks). Missing data were addressed via imputation techniques (Piacentini et al., 2010, Wilhelm et al., 2012).

\section{Statistical Analysis}

To investigate the predictions that PMU severity would decline in those who received CBIT and particularly those who responded to CBIT, as compared to other participants, we conducted $2 \times 2 \times 3$ (treatment condition $\times$ response status $\times$ time) repeated-measures ANOVA tests. For both the child and adult data, Mauchly's tests of sphericity were rejected $\left(X^{2}[2]=12.27, p<0.001 ; X^{2}[2]=16.25, p<\right.$ $0.001)$, so the ANOVAs were interpreted through Greenhouse-Geisser corrected results $(\varepsilon=.897 ; \varepsilon=$ .863). Significant results were further investigated by conducting a one-way ANOVA and bonferroni post-hoc tests comparing the magnitude of PMU reductions across treatment between four groups of participants: participants who received CBIT and responded to treatment, participants who received CBIT and did not respond to treatment, participants who received PST and responded to treatment, and participants who received PST and did not respond to treatment.

To investigate Hypothesis 3, that reductions in PMU severity would mediate the relationship between treatment assignment and outcome, a bootstrapping regression-based technique (Hayes \& Preacher, 2014) was used to measure the strength of the indirect effect of PUTS changes across treatment on the association between treatment assignment and changes in YGTSS scores from baseline to posttreatment. See Figure 1. The SPSS Macro "MEDIATE" was used to perform such analyses (Hayes, 2014). The number of bootstrap samples was set to 5000 , and a $95 \%$ bootstrap percentile confidence interval was used. A significant indirect effect is inferred when zero lies outside of the confidence interval.

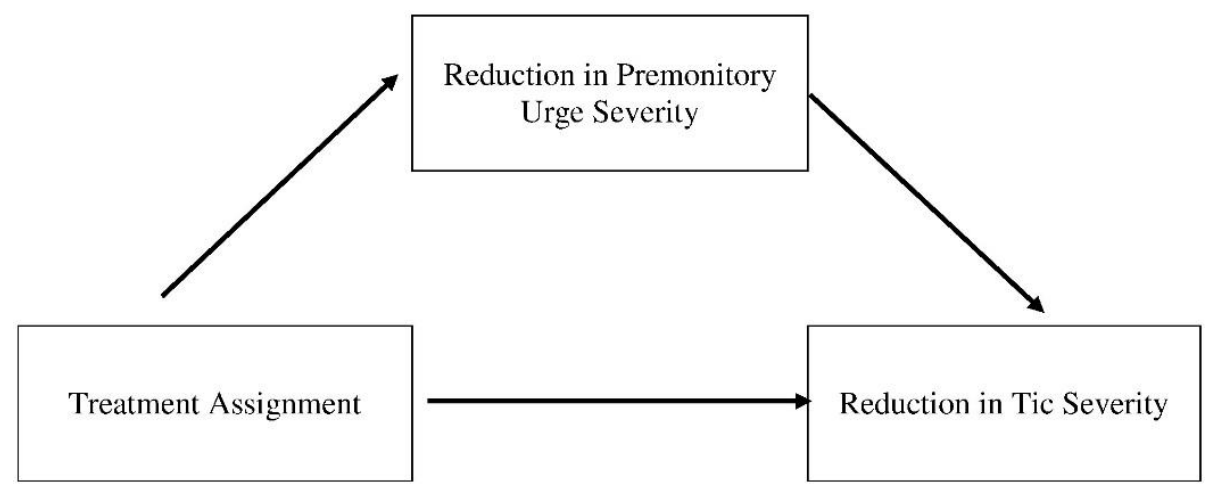

Figure 1. Proposed mediation path in which reductions in premonitory urges exert an indirect effect on the direct relationship between treatment assignment and treatment outcome (reductions in tic severity).

Finally, exploratory analyses investigating the relationship between PMU severity reductions and changes in secondary outcome measures in the clinical trials were conducted using Pearson's correlations. 


\section{Results}

\section{Child Trial}

Results were inconsistent with Hypotheses 1 and 2, in that PMU severity did not decrease over time for either PST or CBIT, nor in those who responded versus those who did not respond to treatment. There were no significant main effects of time, $F(2,204)=1.59, p=0.21$, treatment condition, $F(1,102)=$ $0.52, p=0.47$, or response status, $F(1,102)=0.69, p=0.41$, and there were no significant interactions between time and treatment condition, $F(2,204)=1.33, p=0.27$, time and response status, $F(2,204)=$ $0.89, p=0.41$, or time and treatment and response status, $F(2,204)=0.03, p=0.96$. See Figure 2 . These results are neither consistent with the notion that CBIT results in PMU severity reductions nor with the hypothesis that PMU severity reduction is associated with treatment gains in children who receive behavior therapy for PTDs.

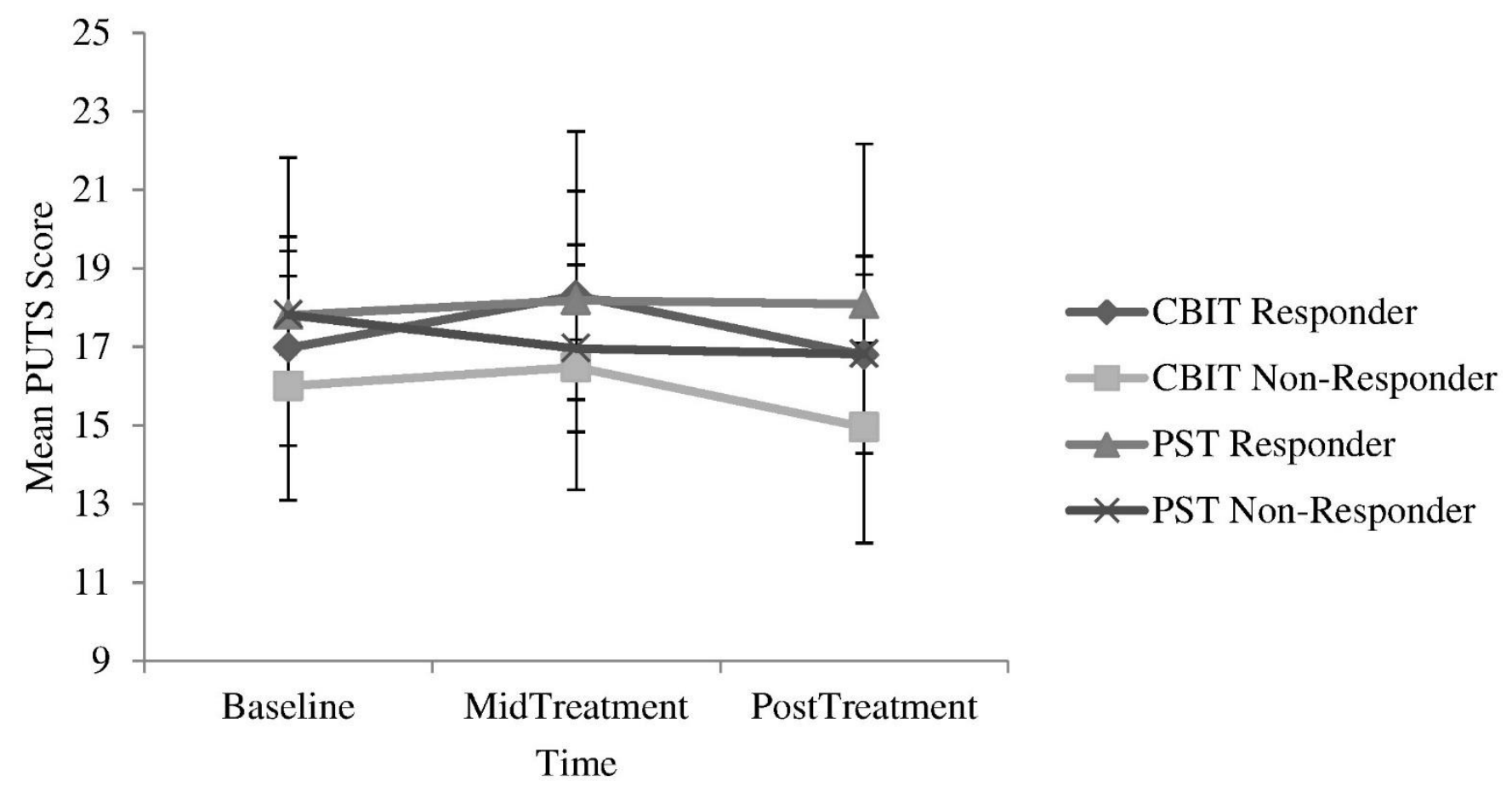

Figure 2. Repeated measures ANOVA of premonitory urge (PMU) trends across treatment in the child trial. Note: $\mathrm{CBIT}=$ Comprehensive Behavioral Intervention for Tics; PST = psychoeducation and supportive psychotherapy; PUTS = Premonitory Urge for Tics Scale. Data points reflect estimated marginal means and $95 \%$ confidence intervals.

When investigating whether changes in PMU mediated the relationship between treatment and outcome, there was a significant direct effect with regard to the relationship between treatment assignment and changes in YGTSS scores, $F(1,104)=12.99, p<0.001$, but there was no significant indirect effect of PMU severity changes (Effect $=0.003$; Lower Level Confidence Interval $=-0.21$, Upper Level Confidence Interval = 0.31). As such, results were inconsistent with Hypothesis 3.

Reductions in PMU severity were not significantly correlated with changes in OCD symptoms, $r(107)=$ $.13, p=.17$, ADHD symptoms, $r(106)=.11, p=.26$, disruptive behavior severity, $r(104)=-.05, p=.62$, or anxiety symptoms as measured by child, $r(106)=.12, p=.21$, or parent, $r(107)=.11, p=.24$. However, 
reductions in PMU severity were significantly correlated with reductions in depression symptoms, $r(103)=.27, p=.006$, and anxiety sensitivity, $r(105)=.27, p=.006$.

\section{Adult Trial}

Results were partially consistent with Hypotheses 1 and 2. PMU severity decreased over time in all participants, but a linear trend of PMU severity reduction was only apparent in those who received CBIT and responded to treatment versus those who did not respond to CBIT and persons who received PST. There was a significant main effect of time, $F(2,190)=4.55, p=0.012, d=0.439$, and no significant main effects of treatment assignment, $F(1,95)=0.003, p=0.96$, or response status, $F(1,95)$ $=0.30, p=0.59$. In addition, there was no significant interaction between time and treatment assignment, $F(2,190)=2.73, p=0.076$, no significant interaction between time and response status, $F(2,190)=2.63, p=0.083$, no significant interaction between treatment assignment and response status, $F(1,95)=3.23, p=0.076$, and a significant three-way interaction between time, treatment assignment, and response status, $F(2,190)=5.16, p=0.009, d=0.468$. See Figure 3. However, although PMU severity generally decreased across the course of treatment in those who responded to CBIT versus showing no such linear trend in other groups, the reduction in PMU severity in those who responded to CBIT was not significantly larger than all other groups. The mean reduction in PUTS scores across treatment in persons who responded to CBIT (22.67 to 19.08; Mean difference $=3.58$, SD $=6.39$ ) was greater than those who did not respond to CBIT (22.69 to 23.17; Mean difference $=-.52$, SD $=.80)(p=.015)$, but it was not greater than persons who received PST and either responded ( 21.75 to 22.75; Mean Difference $=-1.0, \mathrm{SD}=4.32)(p=.51)$ or did not respond to treatment (20.52 to 19.40; Mean difference $=1.33, \mathrm{SD}=4.20)(p=.42)$.

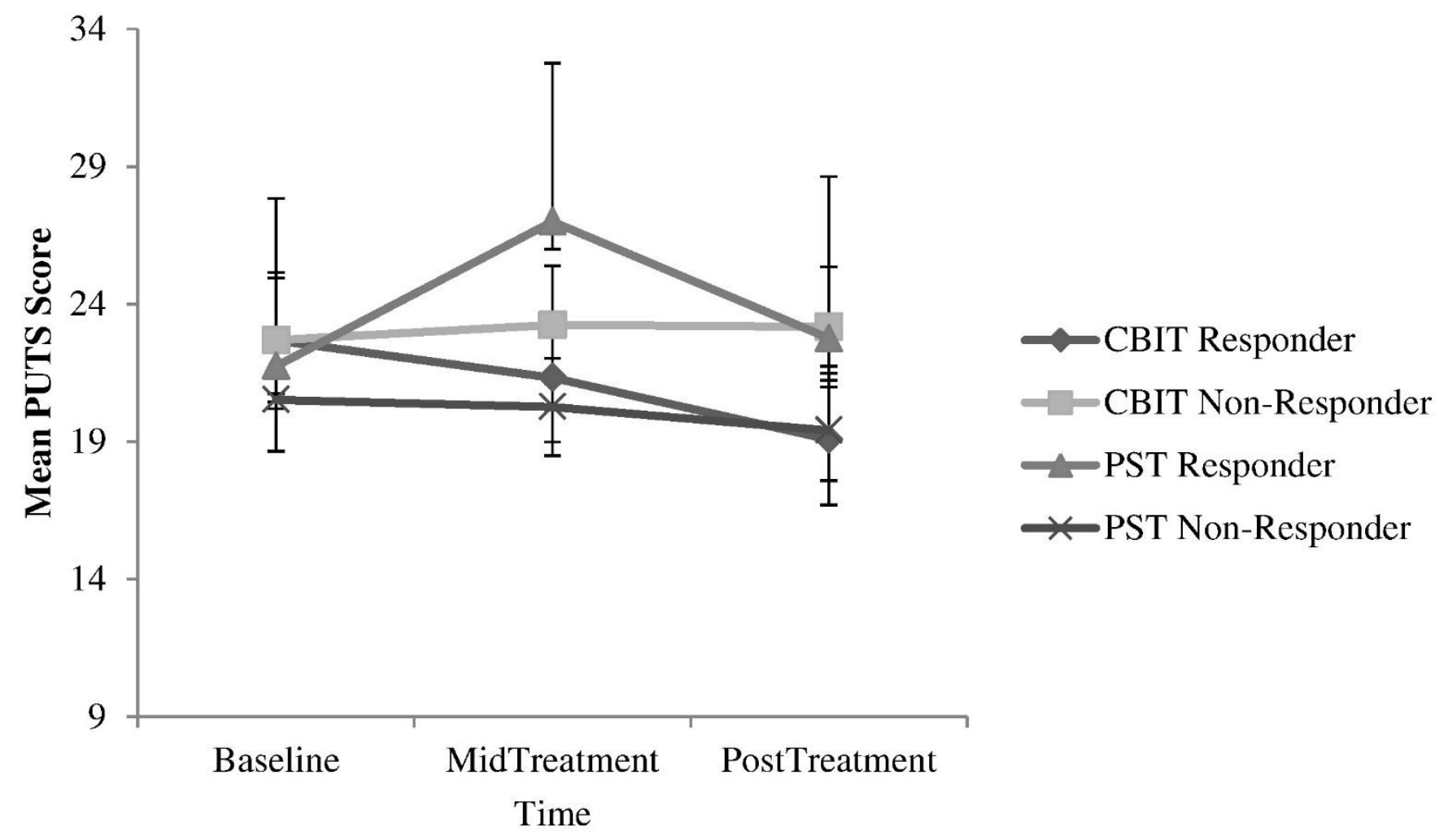

Figure 3. Repeated measures ANOVA of premonitory urge (PMU) trends across treatment in the adult trial. Note: $\mathrm{CBIT}=$ Comprehensive Behavioral Intervention for Tics; $\mathrm{PST}=$ psychoeducation and 
supportive psychotherapy; PUTS = Premonitory Urge for Tics Scale. Data points reflect estimated marginal means and $95 \%$ confidence intervals.

Results failed to support Hypothesis 3, as reductions in PMU severity did not mediate the relationship between treatment and outcome. Although there was a significant direct effect with regard to the relationship between treatment assignment and changes in YGTSS scores, $F(1,101)=15.96, p<0.001$, there was no significant indirect effect of PMU severity changes (Effect $=-0.03$; Lower Level Confidence Interval $=-0.67$, Upper Level Confidence Interval = 0.32).

In exploring the relationship between reductions in PUTS scores and secondary outcome measures, there were no significant correlations between PMU severity reductions and changes in OCD symptoms, $r(104)=-.12, p=.25$, depression, $r(102)=-.06, p=.52$, or anxiety, $r(103)=-.17, p=.09$.

\section{Discussion}

Findings from the current study generally do not support the notion that PMU habituation/reduction in urge severity is a mechanism of change in behavior therapy for PTDs. In children, reductions in PMU severity across treatment were not significantly related to assignment to behavior therapy or response status. Adults who received $\mathrm{CBIT}$ and responded to treatment showed a significant linear trend consistent with PMU severity reductions, but the magnitude of PMU severity reductions in those who responded to CBIT was not significantly greater than in all the other study groups. Moreover, reductions in PMU severity did not mediate the relationship between treatment and outcome in either children or adults, suggesting that even when PMU severity reduction occurs, this process does not drive reductions in tic severity.

Indeed, even though adults who responded to CBIT showed a trend consistent with declining PMU severity as compared to other groups, our findings do not satisfy the necessary criteria to establish that $\mathrm{PMU}$ reductions are mechanisms of change in adult patients. Mediation in clinical trials is evidenced by a main effect of treatment, an interaction between treatment and outcome, as well as a significant indirect effect of the proposed mediator on the relationship between treatment and outcome (Kraemer et al., 2002). Findings from the adult CBIT trial lacked the main effect of treatment but showed an interaction between time, treatment, and outcome. In addition, results showed a main effect of time. Due to the fact that adult patients who received either behavior therapy or supportive therapy showed PMU reductions and because there was no main effect of treatment, we cannot infer that PMU habituation is caused by CBIT specifically.

Present findings necessitate changes in models on the role of PMU habituation in behavior therapy for PTDs. Current models suggest that as tics are suppressed, patients are exposed to the PMU experience, which initially builds and then gradually dissipates; as this cycle is repeated, patients come to habituate to PMUs. As a product of extensive habituation and reduced PMU severity, patients are thought to feel less compelled to tic. Instead, our results contradict this model in several ways. First, it appears that PMU severity reductions can occur in persons whose tic severity improves without explicit training in tic suppression. Perhaps individuals in the adult clinical trial who received PST benefitted from factors such as regression toward the mean or therapeutic common factors, and that as their tic severity declined they experienced concurrent reductions in PMU severity. Additionally, it is possible that PMUs do indeed habituate during CBIT, but PMU habituation is not a large effect that outpaces and drives tic reduction. Results from the adult clinical trial showed that although PMUs did show a declining trend in those who responded to CBIT relative to other groups, the size of PMU severity reductions in those 
who responded CBIT was not significantly larger than persons who both did and did not respond to PST. This would suggest that if PMU habituation does occur in CBIT, it is not a large change that is important for outcomes.

Although study results provide evidence for some degree of PMU severity reductions in adults who responded to behavior therapy, children who underwent treatment did not report significant global PMU reductions. This suggests that there may be important age-based differences in how PMUs are affected by tic treatment. We mentioned earlier that children are less likely to report PMUs, which would suggest that with fewer and less severe PMUs, there may be less room for PMU change across treatment. Visual inspection of Figure 2, Figure 3 provide support for this notion, in that baseline PMU severity was lower in children $(M=17.35)$ than in adults $(M=21.53)$. There is also evidence that the psychometric properties of the PUTS are less satisfactory in young children, which is a limitation to the current study but could also reflect further evidence of age-based differences in PMUs. Indeed, a behavioral model of PMU development provides an expanded explanation of the current findings. Central to this model is the idea that PMU-tic associations are not solidified until years after tics emerge. For instance, researchers have suggested that PMUs emerge and are maintained by not only developmental and neurological factors, but also certain environmental events such as aversive consequences that accompany tics (Capriotti et al., 2013, Himle et al., 2006, Himle et al., 2014, Woods et al., 2005). The underlying neural and somatic correlates of the urge may be present at tic onset, but children may fail to recognize these feelings or experience them as nonaversive. As tics continue to occur and increase in severity, they result in aversive consequences (e.g., pain, embarrassment; Conelea $\&$ Woods, 2008). As a result of these consequences, affected children become more vigilant to sensations that precede tics, and as with any stimulus that signals an impending aversive event, the urges acquire an aversive valence (Woods et al., 2005). Accordingly, as children age and become more attuned to PMUs (possibly around age 10), a conditioned association between PMUs and tics develops and strengthens. Adults, who may be more attuned to this functional relationship, may be more likely to notice that as their tics extinguish and become less frequent, they feel reduced PMUs. By comparison, children who are less attuned to PMUs (i.e., those younger than 10) and have weaker PMU-tic conditioned associations would be expected to show a less clear relationship between tic reductions and PMU reductions.

It is also possible that children with PTDs may have difficulty in differentiating between "true PMUs" and other aversive internal experiences (e.g., sensory underpinnings of negative affect, non-tic somatic symptoms) due to insufficiently developed levels of interoceptive awareness and verbal naming repertoires required to (a) reliably discriminate between between tic-relevant somatosensory experiences and other affective/somatic events, and (b) reliably report on these differences. Although speculative, prior research has found child-reported PUTS scores to correlate with scores on anxiety and depression measures (Eddy and Cavanna, 2013, Rozenman et al., 2015, Steinberg et al., 2009, Woods et al., 2005), and, in the present study, PMU severity reductions in children were associated with improvements in depressive symptoms and anxiety sensitivity.

Findings from the current study have important implications for future research. To date, few alternatives to the habituation model of therapeutic change in behavior therapy for PTD have been offered, but there does appear to be a specific mechanism of action within behavior therapy for PTDs that explains this treatment's unique ability to generate tic reductions. Analyses from various clinical trials indicate that the efficacy of behavior therapy is not the result of common therapeutic factors. Specifically, studies have shown that factors such as tic disclosure (Deckersbach, Rauch, Buhlmann, \& Wilhelm, 2006) and treatment expectancy (Wilhelm et al., 2003) do not account for outcomes in 
behavior therapy for PTDs. Similarly, increased knowledge and validation gained from psychoeducation may produce limited change, but does not appear to account for the majority of clinical change in behavior therapy (Piacentini et al., 2010, Wilhelm et al., 2012). Despite the paucity of clinical research on identified mechanisms of change in behavior therapy of PTDs, there is a relative wealth of neurocognitive research suggesting alternative processes that may be at work during behavior therapy for PTD. Consistent with this view, a study of adults receiving CBIT demonstrated a nuanced relation between changes in left inferior frontal gyrus functioning (known to subserve top-down motor control) and decreases in tic severity across treatment (Deckersbach et al., 2014). This model of increased selfcontrol could be seen as consistent with research on executive control over tics. Laboratory data suggest there is an inverse relation between tic severity and performance on a top-down cognitivemotor control task (Baym, Corbett, Wright, \& Bunge, 2008), and that active tic suppression involves heightened activity in areas involved in top-down control (e.g., the left inferior frontal gyrus; Ganos et al., 2014). A recent review found evidence suggesting that increased control over motor output, which could occur due to repeated tic suppression during development, leads to declining tic severity as affected individuals age (Jackson, Draper, Dyke, Pépés, \& Jackson, 2015). Further, one recent investigation failed to find evidence for habituation across periods of reinforced tic suppression in children (Specht et al., 2013), but the researchers later reported that tics were less likely to occur following severe PMUs that were experienced during reinforced tic suppression periods than following less severe PMUs that were experienced during "free to tic" periods (Specht et al., 2014). This may be seen as indicating that top-down inhibition, manipulated by proxy in this study via the used of reinforcement for tic suppression, led to decreased tic severity in the presence of urges. There are, however, negative findings that contradict the notion that inhibitory control is correlated with improved tic suppression, as a recent study found that improvements in tic severity during CBIT were not associated with performance on a neuropsychological measure of response inhibition (Abramovitch et al., 2017).

In addition to occasioning new directions for future mechanism-focused research, the current study has several immediate implications. These data suggest that, when discussing expectations for therapy, clinicians should indicate to patients that although PMUs may become fewer and less intense over time, treatment really involves learning helpful new ways to manage existing urges. Expectations regarding PMU reduction should be expressed with caution, particularly in child and adolescent cases. Additionally, changes in urge severity across sessions should not be considered an index of treatment progress. Instead, clinicians might consider the patient's ability to suppress tics in the presence of PMUs. A novel approach to managing tics and PMUs in such a way has been tested using mindfulnessbased stress reduction (MBSR; Reese et al., 2015), which teaches patients to respond differently to PMUs by observing and tolerating these experiences without ticcing. In a small-scale uncontrolled trial of MBSR in older adolescents and adults, researchers found that the intervention was well-tolerated and resulted in significant improvements in tic severity and impairment (Reese et al., 2015). Perhaps future research should examine such an alternate model of PMU management in PTDs, focusing less on PMU reduction and more on the functional relationship between PMUs and tics within each individual.

It should be noted that a significant limitation of this study is that the PUTS may not be the perfect instrument for studying nuanced changes in PMU severity over time. Although results of this study support the notion that the PUTS can detect changes in PMUs over time, it could be argued that research on PMU habituation may benefit from assessment on a finer temporal scale (e.g., every 15s), as has been done in laboratory-based tic suppression studies (Capriotti et al., 2014, Capriotti et al., 
2014, Himle et al., 2007, Specht et al., 2013). Moreover, different tics are associated with different PMUs, and CBIT is meant to target only bothersome tics and ignore benign tics (e.g., McGuire et al., 2015). This means that certain PMUs may be affected by CBIT while others remain unchanged, which could limit reductions in the overall PUTS score despite patients feeling that their most bothersome PMUs have reduced significantly. An ideal way in which to maximize the validity of PMU assessment during treatment would be to gather a continuous measure of urge severity from those urges that are tied to targeted tics, measure several different urges simultaneously, and summarize overall PMU severity. Examination of PUTS item content also reveals that the measure could be characterized as more an inventory of different types of urge experiences, rather than a multidimensional assessment of urge frequency, intensity, and resistance to change. Future studies on therapeutic processes in behavior therapy for PTDs may benefit from using assessment measures designed to measure such important constructs, such as the Individualized Premonitory Urge for Tics Scale, which measures PMUs severity on a tic-by-tic basis (McGuire et al., 2016).

\section{Conclusion}

Research on behavior therapy for PTDs is burgeoning, and increasing efforts are being made to understand the processes through which these treatments work. At odds with the dominant habituation model, this study found that although adults who respond to CBIT show PMU severity reductions, children who receive $\mathrm{CBIT}$ do not show PMU severity reductions, and PMU severity reductions do not mediate change. These results suggest that more attention be devoted to processes of change in behavior therapy for PTDs, with specific consideration of alternative models of change.

\section{Conflict of Interest Statement}

Drs. Scahill, Wilhelm, Peterson, Walkup, Piacentini, and Woods receive royalties from Oxford University Press. Drs. Scahill, Wilhelm, Walkup, Piacentini, and Woods receive royalties from Guilford Press. Drs. Scahill and Peterson are on the speaker's bureau for the Tourette Syndrome Association. Drs. Wilhelm, Walkup, and Piacentini have received speaking honoraria from the Tourette Syndrome Association. Drs. Wilhelm and Piacentini have received honoraria from the International Obsessive-Compulsive Disorder Foundation. Drs. Walkup and Piacentini have received grants from the Tourette Syndrome Association. Dr. Scahill has consulted for Roche, Neuren, MedAdvante, Coronado, and Shire. Dr. Wilhelm has received research support in the form of free medication and matching placebo from Forest Laboratories for clinical trials funded by the NIH. Dr. Wilhelm is a presenter for the Massachusetts General Hospital Psychiatry Academy. Dr. Wilhelm has received royalties from Elsevier Publications. Dr. Wilhelm received payment from the Association for Behavioral and Cognitive Therapies for her role as Associate Editor for the Behavior Therapy journal, as well as from John Wiley \& Sons, Inc. for her role as Associate Editor on the Journal Depression \& Anxiety. Dr. Wilhelm has also received salary support from Novartis. Dr. Walkup has received free drugs and matching placebos from the following pharmaceutical companies for National Institute of Mental Health funded studies: Eli Lilly (2003), Pfizer (2007), Abbott (2005). Dr. Walkup was paid for a one time consultation with Shire (2011). Dr. Walkup is a paid speaker for the Center for Disease Control and Prevention outreach education programs; American Academy of Child and Adolescent Psychiatry; and American Psychiatric Association. Dr. Walkup receives grant funding from the Hartwell Foundation and is an unpaid advisor to Anxiety Disorders Association of America, Consumer Reports, and the Trichotillomania Learning 
Center. Dr. Piacentini has received research support from Pfizer and the Pettit Family Foundation. Dr. Woods receives royalties from Springer Press.

\section{References}

Abramovitch et al., 2017 A. Abramovitch, L.S. Hallion, H.E. Reese, D.W. Woods, A. Peterson, J.T. Walkup, ..., T. Deckersbach. Neurocognitive predictors of treatment response to randomized treatment in adults with tic disorders. Progress in Neuro-Psychopharmacology \& Biological Psychiatry, 74 (2017), pp. 9-14.

Abramowitz, 1996 J.S. Abramowitz. Variants of exposure and response prevention in the treatment of Obsessive-Compulsive Disorder: A meta-analysis. Behavior Therapy, 27 (1996), pp. 583-600.

Achenbach and Edelbrock, 1991 T.M. Achenbach, C. Edelbrock. Child Behavior Checklist. University of Vermont, Burlington, VT (1991).

Albin and Mink, 2006 R.L. Albin, J.W. Mink. Recent advances in Tourette syndrome research. Trends in Neurosciences, 29 (3) (2006), pp. 175-182.

American Psychiatric Association, 2013 American Psychiatric Association. Diagnostic and statistical manual of mental disorders (5th ed.), Author, Washington, DC (2013).

Azrin and Nunn, 1973 N.H. Azrin, R.G. Nunn. Habit-reversal: A method of eliminating nervous habits and tics. Behavior Research and Therapy, 11 (4) (1973), pp. 619-628.

Banaschewski et al., 2003 T. Banaschewski, W. Woerner, A. Rothenberger. Premonitory sensory phenomena and suppressibility of tics in Tourette syndrome: Developmental aspects in children and adolescents. Developmental Medicine and Child Neurology, 45 (10) (2003), pp. 700-703.

Bandelow et al., 2006 B. Bandelow, D.S. Baldwin, O.T. Dolberg, D.F. Andersen, D.J. Stein. What is the threshold for symptomatic response and remission for major depressive disorder, panic disorder, social anxiety disorder, and generalized anxiety disorder? Journal of Clinical Psychiatry, 67 (9) (2006), pp. 1428-1434.

Baym et al., 2008 C.L. Baym, B.A. Corbett, S.B. Wright, S.A. Bunge. Neural correlates of tic severity and cognitive control in children with Tourette syndrome. Brain, 131 (2008), pp. 165-179.

Beck et al., 1988 A.T. Beck, N. Epstein, G. Brown, R.A. Steer. An inventory for measuring clinical anxiety: Psychometric properties. Journal of Consulting and Clinical Psychology, 56 (6) (1988), pp. 893-897.

Beck et al., 1988 A.T. Beck, R.A. Steer, M.G. Garbin. Psychometric properties of the Beck Depression Inventory: Twenty-five years of evaluation. Clinical Psychology Review, 8 (1) (1988), pp. 77-100.

Birmaher et al., 1997 B. Birmaher, S. Khetarpal, D. Brent, M. Cully, L. Balach, J. Kaufman, S.M. Neer. The Screen for Child Anxiety Related Emotional Disorders (SCARED): Scale construction and psychometric characteristics. Journal of the American Academy of Child \& Adolescent Psychiatry, 36 (4) (1997), pp. 545-553.

Bliss et al., 1980 J. Bliss, D.J. Cohen, D.X. Freedman. Sensory experiences of Gilles de la Tourette syndrome. Archives of General Psychiatry, 37 (12) (1980), pp. 1343-1347.

Bloch and Leckman, 2009 M.H. Bloch, J.F. Leckman. Clinical course of Tourette syndrome. Journal of Psychosomatic Research, 67 (6) (2009), pp. 497-501. http://dx.doi.org/10.1016/j.jpsychores.2009.09.002

Brandt et al., 2016 V.C. Brandt, C. Beck, V. Sajin, S. Anders, A. Munchau. Convergent validity of the PUTS. Frontiers in Psychiatry, 7 (51) (2016), pp. 1-7. 
Brandt et al., 2016 V.C. Brandt, C. Beck, V. Sajin, M.K. Baaske, T. Baumer, C. Beste, ..., A. Munchau. Temporal relationship between premonitory urges and tics in Gilles de la Tourette syndrome. Cortex, 77 (2016), pp. 24-37.

Caine et al., 1979 E.D. Caine, R.J. Polinsky, R. Kartzinel, M.H. Ebert. The trial use of clozapine for abnormal involuntary movements. American Journal of Psychiatry, 136 (1979), pp. 317-320.

Capriotti et al., 2014 M.R. Capriotti, B.C. Brandt, J.E. Turkel, H.-J. Lee, D.W. Woods. Negative Reinforcement and Premonitory Urges in Youth With Tourette Syndrome: An Experimental Evaluation. Behavior Modification (2014). 0145445514531015. http://dx.doi.org/10.1177/0145445514531015

Capriotti et al., 2013 M.R. Capriotti, F.M. Espil, C.A. Conelea, D.W. Woods. Environmental factors as potential determinants of premonitory urge severity in youth with Tourette syndrome. Journal of Obsessive-Compulsive and Related Disorders, 2 (1) (2013), pp. 37-42.

Capriotti et al., 2014 M.R. Capriotti, M.B. Himle, D.W. Woods. Behavioral treatments for Tourette syndrome. Journal of Obsessive-Compulsive and Related Disorders, 3 (4) (2014), pp. 415-420.

Cohen and Leckman, 1992 A.J. Cohen, J.F. Leckman. Sensory phenomena associated with Gilles de la Tourette's syndrome. Journal of Clinical Psychiatry, 53 (9) (1992), pp. 319-323.

Conelea and Woods, 2008 C.A. Conelea, D.W. Woods. The influence of contextual factors on tic expression in Tourette's syndrome: A review. Journal of Psychosomatic Research, 65 (5) (2008), pp. 487-496. http://dx.doi.org/10.1016/j.jpsychores.2008.04.010

Cook and Blacher, 2007 C.R. Cook, J. Blacher. Evidence-based psychosocial treatments for tic disorders. Clinical Psychology: Science and Practice, 14 (2007), pp. 252-267.

Crossley et al., 2014 E. Crossley, S. Seri, J.S. Stern, M.M. Robertson, A.E. Cavanna. Premonitory urges for tics in adult patients with Tourette syndrome. Brain and Development-Jpm, 36 (1) (2014), pp. 45-50. http://dx.doi.org/10.1016/j.braindev.2012.12.010

Deckersbach et al., 2014 T. Deckersbach, T. Chou, J.C. Britton, L.E. Carlson. Neural correlates of behavior therapy for Tourette' s disorder. Psychiatry Research: Neuroimaging, 224 (3) (2014), pp. 269-274. http://dx.doi.org/10.1016/j.pscychresns.2014.09.003

Deckersbach et al., 2006 T. Deckersbach, S. Rauch, U. Buhlmann, S. Wilhelm. Habit reversal versus supportive psychotherapy in Tourette's disorder: A randomized controlled trial and predictors of treatment response. Behavior Research and Therapy, 44 (8) (2006), pp. 1079-1090.

Eddy and Cavanna, 2013 C.M. Eddy, A.E. Cavanna. Premonitory urges in adults with complicated and uncomplicated Tourette syndrome. Behavior Modification, 38 (2) (2013), pp. 264-275.

Evers and van de Wetering, 1994 R.A. Evers, B.J. van de Wetering. A treatment model for motor tics based on a specific tension-reduction technique. Journal of Behavior Therapy and Experimental Psychiatry, 25 (3) (1994), pp. 255-260.

Friedman-Weieneth et al., 2009 J.L. Friedman-Weieneth, G.L. Doctoroff, E.A. Harvey, L.H. Goldstein. The Disruptive Behavior Rating Scale-Parent version (DBRS-PV) factor analytic structure and validity among young preschool children. Journal of Attention Disorders, 13 (1) (2009), pp. 4255.

Ganos et al., 2014 C. Ganos, U. Kahl, V. Brandt, O. Schunke, T. Bäumer, G. Thomalla, et al. The neural correlates of tic inhibition in Gilles de la Tourette syndrome. Neuropsychologia, 65 (2014), pp. 297-301. http://dx.doi.org/10.1016/j.neuropsychologia.2014.08.007

Goetz and Horn, 2005 C. Goetz, S. Horn. The treatment of tics. Handbook of Tourette's Syndrome and Related Tic and Behavioral Disorders, Marcel Dekker, New York, NY (2005), pp. 411-426. 
Goodman et al., 1989 W.K. Goodman, L.H. Price, S.A. Rasmussen, C. Mazure, R.L. Fleischmann, C.L. Hill, ..., D.S. Charney. The Yale-Brown Obsessive Compulsive Scale: I. Development, use, and reliability. Archives of General Psychiatry, 46 (1989), pp. 1006-1011.

Groth et al., 2017 C. Groth, N.M. Debes, C.U. Rask, T. Lange, L. Skov. Course of Tourette syndrome and comorbidities in a large prospective clinical study. Journal of the American Academy of Child \& Adolescent Psychiatry, 56 (4) (2017), pp. 304-312.

Guy and Bonato, 1970 W. Guy, R.R. Bonato. CGI: Clinical Global Impressions Scale. Manual for the ECDEU Assessment Battery, 1976, US Department of Health, Education, and Welfare, National Institute of Mental Health, Rockville, MD (1970), pp. 217-222.

Hayes, 2014 A.F. Hayes. MEDIATE [Computer software]. Retrieved from http://afhayes.com/spss-sasand-mplus-macros-and-code.html (2014)

Hayes and Preacher, 2014 A.F. Hayes, K.J. Preacher. Statistical mediation analysis with a multicategorical independent variable. British Journal of Mathematical and Statistical Psychology, 67 (2014), pp. 451-470.

Helsel and Matson, 1984 W.J. Helsel, J.L. Matson. The assessment of depression in children: The internal structure of the child depression inventory (CDI). Behavior Research and Therapy, 22 (3) (1984), pp. 289-298.

Himle et al., 2014 M.B. Himle, M.R. Capriotti, L.R. Hayes, K. Ramanujam, L. Scahill, D.G. Sukhodolsky, ..., J. Paicentini. Variables associated with tic exacerbation in children with chronic tic disorders. Behavior Modification, 38 (2) (2014), pp. 163-183.

Himle et al., 2007 M.B. Himle, D.W. Woods, C.A. Conelea, C.C. Bauer, K.A. Rice. Investigating the effects of tic suppression on premonitory urge ratings in children and adolescents with Tourette's syndrome. Behavior Research and Therapy, 45 (12) (2007), pp. 2964-2976. http://dx.doi.org/10.1016/j.brat.2007.08.007

Himle et al., 2006 M.B. Himle, D.W. Woods, J.C. Piacentini, J.T. Walkup. Brief Review of Habit Reversal Training for Tourette Syndrome. Journal of Child Neurology, 21 (8) (2006), pp. 719-725. http://dx.doi.org/10.1177/08830738060210080101

Hoogduin et al., 1997 K. Hoogduin, C. Verdellen, D. Cath. Exposure and Response Prevention in the Treatment of Gilles de la Tourette's Syndrome: Four Case Studies. Clinical Psychology \& Psychotherapy, 4 (2) (1997), pp. 125-135. http://doi.org/10.1002/(SICI)10990879(199706)4:2 < 125::AID-CPP125 > 3.0.CO;2-Z

Houghton et al., 2016 D.C. Houghton, J.R. Alexander, D.W. Woods. The psychosocial impact of tic disorders: Nature and intervention. Journal of Developmental and Physical Disabilities, 28 (2) (2016), pp. 347-365.

Houghton et al., 2014 D.C. Houghton, M.R. Capriotti, C.A. Conelea, D.W. Woods. Sensory phenomena in Tourette syndrome: Their role in symptom formation and treatment. Current Developmental Disorders, 1 (4) (2014), pp. 245-251.

Jackson et al., 2015 G.M. Jackson, A. Draper, K. Dyke, S.E. Pépés, S.R. Jackson. Inhibition, disinhibition, and the control of action in Tourette syndrome. Trends in Cognitive Science, 19 (11) (2015), pp. 655-665.

Kane, 1994 M.J. Kane. Premonitory urges as "attentional tics" in Tourette's syndrome. Journal of the American Academy of Child \& Adolescent Psychiatry, 33 (6) (1994), pp. 805-808.

Kraemer et al., 2002 H.C. Kraemer, G.T. Wilson, C.G. Fairburn, W.S. Agras. Mediators and moderators of treatment effects in randomized clinical trials. Archives of General Psychiatry, 59 (10) (2002), pp. 877-883. 
Kurlan et al., 1989 R. Kurlan, D. Lichter, D. Hewitt. Sensory tics in Tourette's syndrome. Neurology, 39 (5) (1989), p. 731.

Kwak et al., 2003 C. Kwak, K. Dat Vuong, J. Jankovic. Premonitory sensory phenomena in Tourette's syndrome. Movement Disorders, 18 (12) (2003), pp. 1530-1533.

Lang, 1991 A. Lang. Patient perception of tics and other movement disorders. Neurology, 41 (2 Part 1) (1991), p. 223.

Leckman et al., 1989 J.F. Leckman, M.A. Riddle, M.T. Hardin, S.I. Ort, K.L. Swartz, J. Stevenson, D.J. Cohen. The Yale Global Tic Severity Scale: initial testing of a clinician-rated scale of tic severity. Journal of the American Academy of Child \& Adolescent Psychiatry, 28 (4) (1989), pp. 566-573. http://dx.doi.org/10.1097/00004583-198907000-00015

Leckman et al., 1993 J.F. Leckman, D.E. Walker, D.J. Cohen. Premonitory urges in Tourette's syndrome. American Journal of Psychiatry, 150 (1) (1993), pp. 98-102.

Leckman et al., 1994 J.F. Leckman, D.E. Walker, W.K. Goodman, D.L. Pauls, D.J. Cohen. "Just right" perceptions associated with compulsive behavior in Tourette's syndrome. American Journal of Psychiatry, 151 (5) (1994), pp. 675-680.

Leckman et al., 1998 J.F. Leckman, H. Zhang, A. Vitale, F. Lahnin, K. Lynch, C. Bondi, ..., B.S. Peterson. Course of tic severity in Tourette syndrome: The first two decades. Pediatrics, 102 (1) (1998), pp. 14-19.

Leon et al., 1993 A.C. Leon, M.K. Shear, G.L. Klerman, L. Portera, J.F. Rosenbaum, I. Goldenberg. A comparison of symptom determinants of patient and clinician global ratings in patients with panic disorder and depression. Journal of Clinical Psychopharmacology, 13 (5) (1993), pp. 327331.

Leucht and Engel, 2006 S. Leucht, R.R. Engel. The relative sensitivity of the Clinical Global Impressions Scale and the Brief Psychiatric Rating Scale in antipsychotic drug trials. Neuropsychopharmacology, 31 (2) (2006), pp. 406-412.

McGuire et al., 2016 J.F. McGuire, N. McBride, J. Piacentini, C. Johnco, A.B. Lewin, T.K. Murphy, E.A. Storch. The premonitory urge revisited: An individualized premonitory urge for tics scale. Journal of Psychiatric Research, 83 (2016), pp. 176-183.

McGuire et al., 2014 J.F. McGuire, J. Piacentini, E.A. Brennan, A.B. Lewin, T.K. Murphy, B.J. Small, E.A. Storch. A meta-analysis of behavior therapy for Tourette syndrome. Journal of Psychiatric Research, 50 (2014), pp. 106-112.

McGuire et al., 2015 J.F. McGuire, J. Piacentini, L. Scahill, D.W. Woods, R. Villarreal, S. Wilhelm, J. Walkup, A.L. Peterson. Bothersome tics in patient with chronic tic disorders: Characteristics and individualized treatment response to behavior therapy. Behavior Research and Therapy, 70 (2015), pp. 56-63.

Piacentini et al., 2010 J. Piacentini, D.W. Woods, L. Scahill, S. Wilhelm, A.L. Peterson, S. Chang, et al. Behavior therapy for children with tourette disorder. Journal of the American Medical Association, 303 (19) (2010), pp. 1929-1937.

Reese et al., 2014 H.E. Reese, L. Scahill, A.L. Peterson, K. Crowe, D.W. Woods, J. Piacentini, et al. The premonitory urge to tic: Measurement, characteristics, and correlates in older adolescents and adults. Behavior Therapy, 45 (2) (2014), pp. 177-186.

http://dx.doi.org/10.1016/j.beth.2013.09.002

Reese et al., 2015 H.E. Reese, Z. Vallejo, J. Rasmussen, K. Crowe, E. Rosenfield, S. Wilhelm. Mindfulness-based stress reduction for Tourette syndrome and chronic tic disorder: A pilot study. Journal of Psychosomatic Research, 78 (3) (2015), pp. 293-298. 
Rozenman et al., 2015 R. Rozenman, O. Johnson, S. Chang, D. Woods, J. Walkup, S. Wilhelm, A. Peterson, L. Scahill, J. Piacentini. Relationships between Premonitory Urge and Anxiety in Youth with Chronic Tic Disorders. Children's Health Care, 44 (2015), pp. 235-248.

Scahill et al., 1997 L. Scahill, M.A. Riddle, M. McSwiggin-Hardin, S.I. Ort, R.A. King, W.K. Goodman, D. Cicchetti. Children's Yale-Brown Obsessive Compulsive scale: Reliability and validity. Journal of the American Academy of Child \& Adolescent Psychiatry, 36 (6) (1997), pp. 844-852.

Silverman et al., 2010 W.K. Silverman, W. Fleisig, B. Rabian, R.A. Peterson. Childhood anxiety sensitivity index. Journal of Clinical Child Psychology, 20 (2) (2010), pp. 162-168.

Specht et al., 2014 M.W. Specht, C.M. Nicotra, L.M. Kelly, D.W. Woods, E.J. Ricketts, C. Perry-Parrish, et al. A comparison of urge intensity and the probability of tic completion during tic freely and tic suppression conditions. Behavior Modification, 38 (2) (2014), pp. 297-318.

http://dx.doi.org/10.1177/0145445514537059

Specht et al., 2013 M.W. Specht, D.W. Woods, C.M. Nicotra, L.M. Kelly, E.J. Ricketts, C.A. Conelea, et al. Effects of tic suppression: Ability to suppress, rebound, negative reinforcement, and habituation to the premonitory urge. Behavior Research and Therapy, 51 (1) (2013), pp. 24-30. http://dx.doi.org/10.1016/j.brat.2012.09.009

Specht et al., 2011 M.W. Specht, D.W. Woods, J. Piacentini, L. Scahill, S. Wilhelm, A.L. Peterson, ..., B.A. Buzella. Clinical characteristics of children and adolescents with a primary tic disorder. Journal of Developmental and Physical Disabilities, 23 (1) (2011), pp. 15-31.

Spielmans and McFall, 2006 G.I. Spielmans, J.P. McFall. A comparative meta-analysis of the Clinical Global Impressions change in antidepressant trials. Journal of Nervous and Mental Disease, 194 (11) (2006), pp. 845-854.

Steinberg et al., 2009 T. Steinberg, S. Shmuel Baruch, A. Harush, R. Dar, D. Woods, J. Piacentini, A. Apter. Tic disorders and the premonitory urge. Journal of Neural Transmission, 117 (2) (2009), pp. 277-284. http://dx.doi.org/10.1007/s00702-009-0353-3

Ventura et al., 2007 J. Ventura, A. Cienfuegos, O. Boxer, R. Bilder. Clinical global impression of cognition in schizophrenia (CGI-CogS): Reliability and validity of a co-primary measure of cognition. Schizophrenia Research, 106 (1) (2007), pp. 59-69.

Verdellen et al., 2008 C.W.J. Verdellen, C.A.L. Hoogduin, B.S. Kato, G.P.J. Keijsers, D.C. Cath, H.B. Hoijtink. Habituation of premonitory sensations during exposure and response prevention treatment in Tourette's Syndrome. Behavior Modification, 32 (2) (2008), pp. 215-227. http://dx.doi.org/10.1177/0145445507309020

Verdellen et al., 2004 C.W.J. Verdellen, G.P.J. Keijsers, D.C. Cath, C.A.L. Hoogduin. Exposure with response prevention versus habit reversal in Tourette's syndrome: A controlled study. Behaviour Research and Therapy, 42 (5) (2004), pp. 501-511.

Wilhelm et al., 2003 S. Wilhelm, T. Deckersbach, B.J. Coffey, A. Bohne, A.L. Peterson, L. Baer. Habit reversal versus supportive psychotherapy for Tourette's disorder: A randomized controlled trial. American Journal of Psychiatry, 160 (6) (2003), pp. 1175-1177.

Wilhelm et al., 2012 S. Wilhelm, A.L. Peterson, J. Piacentini, D.W. Woods, T. Deckersbach, D.G. Sukhodolsky, ..., L. Scahill. Randomized trial of behavior therapy for adults with Tourette syndrome. Archives of General Psychiatry, 69 (8) (2012), pp. 795-803.

Woods and Himle, 2004 D.W. Woods, M.B. Himle. Creating tic suppression: Comparing the effects of verbal instruction to differential reinforcement. Journal of Applied Behavior Analysis, 37 (3) (2004), pp. 417-420.

Woods et al., 2000 D.W. Woods, S.S. Hook, D.F. Spellman, P.C. Friman. Case study: Exposure and response prevention for an adolescent with Tourette's syndrome and OCD. Journal of the 
American Academy of Child \& Adolescent Psychiatry, 39 (7) (2000), pp. 904-907.

http://dx.doi.org/10.1097/00004583-200007000-00020

Woods et al., 2008 D.W. Woods, J. Piacentini, S. Chang, T. Deckersbach, G. Ginsburg, A. Peterson, et al. Managing Tourette Syndrome: A behavioral intervention for children and adults therapist guide. Oxford University Press, New York, NY (2008).

Woods et al., 2005 D.W. Woods, J. Piacentini, M.B. Himle, S. Chang. Premonitory Urge for Tics Scale (PUTS): Initial psychometric results and examination of the premonitory urge phenomenon in youths with Tic disorders. Journal of Developmental \& Behavioral Pediatrics, 26 (6) (2005), pp. 397-403.

Zaider et al., 2003 T.I. Zaider, R.G. Heimberg, D.M. Fresco, F.R. Schneier, M.R. Liebowitz. Evaluation of the clinical global impression scale among individuals with social anxiety disorder. Psychological Medicine, 33 (4) (2003), pp. 611-622.

iThis research was supported by National Institute of Mental Health (NIMH) Grants R01MH070802 (Dr. Piacentini), 5R01MH069877 (Dr. Wilhelm), R01MH069874 (Dr. Scahill), and R01MH069875 (Dr. Peterson) with subcontracts to Drs. Walkup and Woods. The funding agency played no role in the study design; collection; analysis or interpretation of data; the writing of this article; or the decision to submit the article for publication. We thank Julie Collins and Joel Williams for their role in providing editorial assistance in the preparation of this manuscript.

败 Clinical trial registration information: U.S. National Institutes of Health human subject's trial forum (ClinicalTrials.gov; \#NCT00218777, \#NCT00231985) 